\title{
Periodic Solution for A Stochastic Non-autonomous Predator-prey Model with Crowley-Martin Function Response
}

\author{
Hongliang Chen and Xiaoping $\mathrm{Li}^{*}$ \\ College of Science, Hunan Agricultural University, Changsha, Hunan 410128, China \\ ${ }^{*}$ Corresponding author
}

\begin{abstract}
In this paper, we investigate a stochastic nonautonomous Crowley-Martin predator-prey model. For the model, we first prove the existence and uniqueness of the global positive solution. For the periodic model, we prove that the existence of a positive periodic solution by using Khasminskii's method and constructing appropriate Lyapunov function.
\end{abstract}

Keywords-stochastic Crowley-Martin predator-prey model; global positive solution; positive periodic solution

\section{INTRODUCTION}

The model of predator and prey play an important role in population theory, and many researchers have paid their attention to the dynamical behavior of predator-prey model. Lotka-Volterra [1] is the earliest predator-prey model, differential equations as following

$$
\left\{\begin{array}{l}
d x(t)=x(t)(r-a x(t)-f y(t)) d t \\
d y(t)=y(t)(-d+h x(t)) d t .
\end{array}\right.
$$

As a predator-prey model, it is common phenomenon that predators have to search, share and compete for food. Therefore, the functional response is one of the significant elements which have influence on the relationship between predator and prey [2]. For better description predator-prey phenomenon, we will investigate Crowley-Martin functional response [3]. So we obtain the following the deterministic predator-prey model with Crowley-Martin functional response

$$
\left\{\begin{array}{l}
d x(t)=x(t)\left(r(t)-a(t) x(t)-\frac{b y(t)}{1+c x(t)+e y(t)+c e x(t) y(t)}\right) d t \\
d y(t)=y(t)\left(-d(t)+\frac{m b x(t)}{1+c x(t)+e y(t)+\operatorname{cex}(t) y(t)}\right) d t
\end{array}\right.
$$

where $x(t), y(t)$ represent the population densities of prey and predator at time $t$, respectively. $r(t)$ and $d(t)$ are the respective intrinsic growth rates of the prey and predator, $a(t)$ is the density-dependent coefficients of prey specie. $b(t)$ is the capturing rate of predator, $m(t)$ denotes the rate of conversion of nutrients into the production, $c(t)$ and $e(t)$ describe the effects of handling time and the magnitude of interference among predators.

However, in the real world, the species are disturbed by environmental noises. We consider the model (2) underlying stochastic perturbation. Assume the intrinsic growth rates $r(t), d(t)$ of the prey and predator are disturbed with

$$
r(t) \rightarrow r(t)+\alpha(t) \dot{B}_{1}(t), d(t) \rightarrow d(t)+\beta(t) \dot{B}_{2}(t)
$$

where $B_{1}, B_{2}$ are standard Brownian motion, $\alpha(t), \beta(t)$ denote the intensity of the white noises. Then we obtain the following stochastic model

$$
\left\{\begin{aligned}
d x(t) & =x(t)\left(r(t)-a(t) x(t)-\frac{b(t) y(t)}{1+c(t) x(t)+e(t) y(t)+c(t) e(t) x(t) y(t)}\right) d t \\
& +\alpha(t) x(t) d B_{1}(t), \\
d y(t) & =y(t)\left(-d(t)+\frac{m(t) b(t) x(t)}{1+c(t) x(t)+e(t) y(t)+c(t) e(t) x(t) y(t)}\right) d t \\
& -\beta(t) y(t) d B_{2}(t),
\end{aligned}\right.
$$

we assume that solution of (3) satisfy the initial condition

$$
x(0)=x_{0}>0, y(0)=y_{0}>0
$$

And the parameters function $r(t), a(t), d(t), b(t)$, $m(t), c(t), e(t), \alpha(t)$ and $\beta(t)$ are positive and continuous $\theta$-periodic functions.

Periodic solutions have been a central concept in the theory of the dynamical system, and periodic behaviors arises naturally in many real world problems. In recent years, several authors have studied some stochastic population models with Crowley-Martin functional response(see[4-5]). But so far, still 
no scholar has investigated the existence of periodic solutions of stochastic population models with Crowley-Martin functional response.

The rest of this paper is arranged as follow. In section 2, we give some basic definitions and lemmas for our succeeding proof. In section 3 , we discuss the existence and uniqueness of the global positive solution. In section 4, we discuss the existence of a positive periodic solution. Finally, a brief conclusion of this paper. incorporating the applicable criteria that follow.

\section{PRELIMINARIES}

Throughout the article, we always use the following notations. Let $(\Omega, F, \mathrm{P})$ be a complete probability space with a filtration $\left\{F_{t}\right\}_{t \geq 0}$ satisfying the usual conditions (i.e. it is right continuous and $F_{0}$ contains all $P$-null sets). If $f(t)$ is a continuous and bounded function on $[0,+\infty]$, we define

$$
\begin{gathered}
\langle f\rangle_{\theta}=\frac{1}{\theta} \int_{0}^{\theta} f(s) d s, \quad \theta>0 \\
f^{u}=\sup _{t \in[0,+\infty]} f(t), f^{l}=\inf _{t \in[0,+\infty]} f(t)
\end{gathered}
$$

Next, we will present a basic definition and introduce a lemma about periodic Markov process.

Definition 2.1[6]. A stochastic process $\xi(t)=\xi(t, \omega)$ $(-\infty<t<+\infty)$ is said to be periodic $\theta$ with period if for every finite sequence of numbers $t_{1}, t_{2}, \cdots, t_{n}$ the joint distribution of random variables $\xi\left(t_{1}+h\right), \cdots, \xi\left(t_{n}+h\right)$ is independent of $h$, where $h=k \theta, k= \pm 1, \pm 2, \cdots$.

Remark 2.1.In[6], it is showed that a Markov process $x(t)$ is $\theta$ periodic if and only if its transition probability function is $\theta$ periodic and the function $\mathrm{P}_{0}(t, A)=\mathrm{P}\{X(t) \in A\}$ satisfies the equation

$\mathrm{P}_{0}(s, A)=\int_{\mathbb{R}^{d}} \mathrm{P}_{0}(s, d x) \mathrm{P}(s, x, s+\theta, A) \equiv \mathrm{P}_{0}(s+\theta, A)$,

where $A \in \mathrm{B}$ and $\mathrm{B}$ is Borel $\sigma$-algebra.

Consider the follow equation

$$
X(t)=X\left(t_{0}\right)+\int_{t_{0}}^{t} b(s, X(s)) d s+\sum_{r=1}^{k} \int_{t_{0}}^{t} \sigma_{r}(s, X(s)) d B_{r}(s), X \in \mathbb{R}^{d}
$$

Lemma 2.1[6]. Suppose that the coefficient of (4) is $\theta$ periodic in $t$ and satisfies the condition

$$
\begin{gathered}
|b(s, x)-b(s, y)|+\sum_{r=1}^{k}\left|\sigma_{r}(s, x)-\sigma_{r}(s, y)\right| \leq B|x-y| \\
|b(s, x)|+\sum_{r=1}^{k}\left|\sigma_{r}(s, x)\right| \leq B(1+|x|),
\end{gathered}
$$

in every cylinder $I \times U$, where B is a constant; and suppose further that there exists a function $V(t, x) \in C^{2}$ in $\mathbb{R}^{d}$ which is $\theta$-periodic in $t$, and satisfies the following conditions

$$
\inf _{|x|>\mathbb{R}} V(t, x) \rightarrow \infty \text { as } \mathbb{R} \rightarrow \infty
$$

and

$$
L V(t, x) \leq-1 \text { on the outside of some compact set, }
$$

where the operator $L$ is given by

$$
L=\frac{\partial}{\partial t}+\sum_{i=1}^{d} b_{i}(t, x) \frac{\partial}{\partial x_{i}}+\frac{1}{2} \sum_{i, j=1}^{d} a_{i j}(t, x) \frac{\partial^{2}}{\partial x_{i} \partial x_{j}}, a_{i j}=\sum_{r=1}^{k} \sigma_{r}^{i}(t, x) \sigma_{r}^{j}(t, x)
$$

Then there exists a solution of (4) which is a $\theta$-periodic Markov process.

\section{EXISTENCE AND UNIQUENESS OF THE GLOBAL POSITIVE SOLUTION}

In the section, we will present the results on the existence and uniqueness of the global positive solution.

Theorem3.1. For any given initial value $X_{0}=\left(x_{0}, y_{0}\right) \in \mathbb{R}_{+}^{2}$, model (3) has a unique solution $(x(t), y(t))$ exists on $t \geq 0$, and the solution will remain in $\mathbb{R}_{+}^{2}$ with probability one.

Proof. Obviously, the coefficients of (3) are locally continuous. In Mao [7], for any given initial value $X_{0} \in \mathbb{R}_{+}^{2}$, there exists a unique maximal local solution $X(t)=(x(t), y(t))$ on $t \in\left[0, \tau_{e}\right)$, where $t \in\left[0, \tau_{e}\right)$ is the explosion time. In order to show that the solution is global, we shall to show $\tau_{e}=\infty$ a.s. Let $\eta_{0}>0$ be sufficiently large so that $x_{0}, y_{0} \in\left[1 / n_{0}, n_{0}\right]$. For every integer $n>n_{0}$, define the stopping time $\tau_{n}=\inf \left\{t \in\left[0, \tau_{e}\right): x(t) \notin(1 / n, n)\right.$ or $\left.y(t) \notin(1 / n, n)\right\}$.

And we set inf $\phi=\infty$ ( $\phi$ as usual denote the empty set). It is obviously that $\tau_{n}$ is increasing as $n \rightarrow \infty$. 
Denote $\tau_{e}=\lim _{n \rightarrow \infty} \tau_{n}$, thus $\tau_{\infty} \leq \tau_{e}$. Hence, we only need to prove that $\tau_{\infty}=\infty$ a.s. If this statement is not true, there will exist a pair of constants $T>0$ an $\varepsilon \in(0,1)$ such that $\mathrm{P}\left\{\tau_{\infty} \leq \mathrm{T}\right\}>\varepsilon$. Then there exists an integer $n_{1}>n_{0}$ such that

$$
\mathrm{P}\left\{\tau_{n} \leq \mathrm{T}\right\}>\varepsilon \text {, for all } n \geq n_{1} \text {. }
$$

Define the $C^{2}$ function : as follows

$$
V(x, y)=(x-1-\ln x)+(y-1-\ln y)
$$

By the Itô's formula, we have

$$
\begin{aligned}
d V(x, y) & =V_{x} d x+\frac{1}{2} V_{x x}(d x)^{2}+V_{y} d y+\frac{1}{2} V_{y y}(d y)^{2} \\
& =x\left(r-a x-\frac{b y}{1+c x+e y+c e x y}\right) d t+\alpha(x-1) d B_{1}(t) \\
& +y\left(-d+\frac{m b x}{1+c x+e y+c e x y}\right) d t-\left(-d+\frac{m b x}{1+c x+e y+c e x y}-\frac{1}{2} \beta^{2}\right) d t \\
& -\beta(y-1) d B_{2}(t)-\left(r-a x-\frac{b y}{1+c x+e y+c e x y}-\frac{1}{2} \alpha^{2}\right) d t \\
& =L V(x, y) d t+\alpha(x-1) d B_{1}(t)-\beta(y-1) d B_{2}(t)
\end{aligned}
$$

Here

$$
\begin{aligned}
L V(x, y) & \leq-a x^{2}+(r+a) x+\frac{b y}{1+c x+e y+c e x y}-r-d y+\frac{m b x y}{1+c x+e y+c e x y}+d \\
& \leq-a^{l} x^{2}+\left(r^{u}+a^{u}\right) x+\frac{b^{u}}{e^{l}}-d^{l} y+\frac{m^{u} b^{u}}{c^{l} e^{l}}-r^{l}+d^{u} \\
& \leq G
\end{aligned}
$$

where $G$ is a constant.

Integrating both sides of inequality (9) from 0 to $\tau_{n} \wedge T$ $\left(\tau_{n} \wedge T=\min \left\{\tau_{n}, T\right\}\right)$, and taking expectation of sides, we obtain

$$
\begin{aligned}
E V\left(x\left(\tau_{n} \wedge T\right), y\left(\tau_{n} \wedge T\right)\right) & \leq V\left(x_{0}, y_{0}\right)+G E\left(\tau_{n} \wedge T\right) \\
& \leq V\left(x_{0}, y_{0}\right)+G T
\end{aligned}
$$

Set $\Omega_{h}=\left\{\tau_{n} \leq \mathrm{T}\right\}$ for $n \geq n_{1}$, due to (7), we have $\mathrm{P}\left\{\tau_{n} \leq \mathrm{T}\right\}>\varepsilon$. For each $\omega \in \Omega_{n}$, there exists at least one of $x\left(\tau_{n}, \omega\right), y\left(\tau_{n}, \omega\right)$ equaling to $n$ or $1 / n$. Hence $V\left(x\left(\tau_{n}, \omega\right), y\left(\tau_{n}, \omega\right)\right) \quad$ is no less than $n-1-\ln n$ or $1 / n-1+\ln n$. That is
$V\left(x\left(\tau_{n}, \omega\right), y\left(\tau_{n}, \omega\right)\right) \geq \min \{n-1-\ln n, 1 / n-1+\ln n\}$

It follows from (10) that

$$
\begin{aligned}
V\left(x_{0}, y_{0}\right)+G T & \geq E\left[1_{\Omega}(\omega) V(x(\omega), y(\omega))\right] \geq P\left(\Omega_{n}\right) \min \left\{n-1-\ln n, \frac{1}{n}-1+\ln n\right\} \\
& \geq \varepsilon \min \left\{n-1-\ln n, \frac{1}{n}-1+\ln n\right\}
\end{aligned}
$$

Here $1_{\Omega}$ is the indicator function of $\Omega_{n}$. Letting $n \rightarrow \infty$ get the contradiction $+\infty>V\left(x_{0}, y_{0}\right)+G T>+\infty$.

Hence, we have $\tau_{\infty}=\infty$ a.s. This completes the proof.

\section{EXISTENCE OF THE POSITIVE PERIODIC SOLUTION}

In this section, we shall investigate the sufficient conditions for the existence of the periodic solution to stochastic model (3).

Theorem 4.1. If $\lambda=\frac{1}{\theta} \int_{0}^{\theta} d(t)+\frac{\beta^{2}(t)}{2}-\left(r(t)-\frac{\alpha^{2}(t)}{2}\right) d t>0$, then for any initial value $X_{0}=\left(x_{0}, y_{0}\right) \in \mathbb{R}_{+}^{2}$, model (3) exists one positive $\theta$-periodic solution.

Proof. According to the proof of Theorem 3.1, we can obtain that for any initial value $\left(x_{0}, y_{0}\right) \in \mathbb{R}_{+}^{2}$, the model (3) has a unique global positive solution. We only need to verify the conditions (5) and (6) hold.

Construct a $C^{2}$ function $V(t, x, y):[0,+\infty) \times \mathbb{R}_{+}^{2} \rightarrow \mathbb{R}$

$$
\begin{aligned}
V(t, x, y) & =C(\ln x+\ln y)+(x+y)^{2} / 2+C W(t) \\
& =V_{1}(t, x, y)+V_{2}(t, x, y)+V_{3}(t, x, y)
\end{aligned}
$$

where $C>0$ will be determined later and $W(t)$ satisfies

$$
\begin{aligned}
W^{\prime}(t) & =-\frac{1}{\theta} \int_{0}^{\theta} d(t)+\frac{\beta^{2}(t)}{2}-\left(r(t)-\frac{\alpha^{2}(t)}{2}\right) d t+d(t)+\frac{\beta^{2}(t)}{2}-\left(r(t)-\frac{\alpha^{2}(t)}{2}\right) \\
& =-\lambda+d(t)+\frac{\beta^{2}(t)}{2}-\left(r(t)-\frac{\alpha^{2}(t)}{2}\right)
\end{aligned}
$$

It is easy to show that $W(t)$ is a $\theta$-periodic functions. In fact integrating (12) from $t$ to $t+\theta$, we obtain

$$
\begin{aligned}
W(t+\theta)-W(t) & =\int_{t}^{t+\theta} W^{\prime}(s) d s=-\int_{0}^{\theta} d(s)+\frac{\beta^{2}(s)}{2}-\left(r(t)-\frac{\alpha^{2}(t)}{2}\right) d s \\
& +\int_{t}^{t+\theta} d(s)+\frac{\beta^{2}(s)}{2}-\left(r(s)-\frac{\alpha^{2}(s)}{2}\right) d s
\end{aligned}
$$$$
=0
$$ 
In order to confirm the condition (5) of Lemma 2.1, we only need to show that

$$
\inf _{(t, x, y) \in[0,+\infty) \times \mathbb{R}_{+}^{2} \backslash U_{k}} V(t, x, y) \rightarrow \infty \alpha \sigma \quad \mathbb{R} \rightarrow \infty
$$

Where $U_{k}=(1 / k, k) \times(1 / k, k)$. All the coefficients of the quadratic term in $V(t, x, y)$ are positive, thus Condition (5) of Lemma 2.1 is satisfied.

Next, we will verify the Condition (6) of Lemma 2.1. By the Itô's formula, we have

$$
\begin{aligned}
L V_{1}(t, x, y)= & r(t)-a(t) x(t)-\frac{b y}{1+c x+e y+c e x y}-\frac{1}{2} \alpha^{2}(t) \\
& -d(t)+\frac{m b x}{1+c x+e y+c e x y}-\frac{1}{2} \beta^{2}(t) \\
& \leq r(t)-\frac{1}{2} \alpha^{2}(t)-d(t)-\frac{1}{2} \beta^{2}(t)
\end{aligned}
$$

Combining (12) and (13), we get

$$
L\left(V_{1}+V_{3}\right) \leq-C \lambda
$$

Moreover, we have

$$
\begin{aligned}
L V_{2}(t, x, y)= & (x+y)\left(r(t) x-a(t) x^{2}-\frac{b x y}{1+c x+e y+c e x y}-d(t) y+\frac{m b x y}{1+c x+e y+c e x y}\right) \\
& +\frac{1}{2} \alpha^{2}(t) x^{2}+\frac{1}{2} \beta^{2}(t) y^{2} \\
\leq & -a(t) x^{3}+\left(r(t)+\frac{1}{2} \alpha^{2}(t)\right) x^{2}+\left(-d(t)+\frac{1}{2} \beta^{2}(t)\right) y^{2}+(r(t)-d(t)) x y \\
\leq & -a^{l} x^{3}+\left(r^{u}+\frac{1}{2}\left(\alpha^{2}\right)^{u}\right) x^{2}+\left(-d^{l}+\frac{1}{2}\left(\beta^{2}\right)^{u}\right) y^{2}+\left(r^{u}-d^{l}\right) x y
\end{aligned}
$$

By (14) and (15), we can obtain

$$
\begin{aligned}
L V(t, x, y) \leq & -C \lambda-a^{l} x^{3}+\left(r^{u}+\frac{1}{2}\left(\alpha^{2}\right)^{u}\right) x^{2}+\left(-d^{l}+\frac{1}{2}\left(\beta^{2}\right)^{u}\right) y^{2} \\
& +\left(r^{u}-d^{l}\right) x y \\
& =-C \lambda-\frac{1}{2} a^{l} x^{3}-d^{l} y^{2}+Q(x, y)
\end{aligned}
$$

where

$$
\begin{aligned}
& Q(x, y)=-\frac{1}{2} a^{l} x^{3}+\left(r^{u}+\frac{1}{2}\left(\alpha^{2}\right)^{u}\right) x^{2}+\frac{1}{2}\left(\beta^{2}\right)^{u} y^{2}+\left(r^{u}-d^{l}\right) x y, \\
& \text { obviously } C \lambda \geq 2 \text {. And }
\end{aligned}
$$

$$
C=2 / \lambda \max \{1, \sup Q\}
$$

Choose a sufficiently small number $\mathcal{E}$ such that

$$
\begin{aligned}
& -C \lambda-a^{l} / 2 e^{3}+Q_{\text {sup }} \leq-1, \\
& -C \lambda-d^{l} / e^{2}+Q_{\text {sup }} \leq-1 .
\end{aligned}
$$

We consider a bounded open set

$$
D_{e}=\left\{(x, y) \in R_{+}^{2}: e \leq x \leq 1 / e, e \leq y \leq 1 / e\right\}
$$

Define

$$
\begin{gathered}
D_{e}^{1}=\left\{(x, y) \in R_{+}^{2}: 0<x<e\right\}, D_{e}^{2}=\left\{(x, y) \in R_{+}^{2}: 0<y<e\right\}, \\
D_{e}^{3}=\left\{(x, y) \in R_{+}^{2}: x>1 / e\right\} D_{e}^{3}=\left\{(x, y) \in R_{+}^{2}: y>1 / e\right\} .
\end{gathered}
$$

Obviously, $D_{e}^{C}=D_{e}^{1} \cup D_{e}^{2} \cup D_{e}^{3} \cup D_{e}^{4}$. Next, we will show that

$$
L V(t, x, y) \leq-1 \text { ov }[0,+\infty) \times D_{e}^{C}
$$

Case 1. If $(t, x, y) \in[0,+\infty) \times D_{e}^{1} \cup D_{e}^{2}$, we have by (16) and (17)

$$
L V(t, x, y) \leq-C \lambda+Q(x, y) \leq-C \lambda+\frac{C \lambda}{2} \leq-1 .
$$

Case 2. If $(t, x, y) \in[0,+\infty) \times D_{e}^{1} \cup D_{e}^{2}$, we have by (16) and (18)

$$
L V(t, x, y) \leq-C \lambda-\frac{1}{2} a^{l} x^{3}-d y^{2}+Q(x, y) \leq-C \lambda-\frac{a^{l}}{2 e^{3}}+Q_{\text {sup }} \leq-1 .
$$

Case 3. If $(t, x, y) \in[0,+\infty) \times D_{e}^{1} \cup D_{e}^{2}$, we have by (16) and (19)

$$
L V(t, x, y) \leq-C \lambda-\frac{1}{2} a^{l} x^{3}-d y^{2}+Q(x, y) \leq-C \lambda-\frac{d^{l}}{e^{2}}+Q_{\text {sup }} \leq-1 .
$$

Summarize the above, we can obtain

$$
L V(t, x, y) \leq-1, \phi \text { о } \alpha \nu \psi(t, x, y) \in[0,+\infty) \times D_{e}^{C}
$$


Thus, the Condition (6) of Lemma 2.1 is satisfied. Therefore, model (3) has a positive $\theta$-periodic solution. The proof is completed.

\section{CONCLUSION}

This paper is concerned with the dynamics of a stochastic non-autonomous Crowley-Martin predator-prey model. According to the proof of Lemma2.1, the linear growth condition is used to guarantee the existence and uniqueness of the global positive solution of model (3). Thus, we prove that the existence of the global positive solution for guaranteed have solution of succeeding proof in section 3 .

\section{REFERENCES}

[1] R.M. May. Stability and Complexity in Model Ecosystems, Princeton University Press, Princeton, 1973.

[2] X.Q. Liu et al. Zhong. Asymptotic properties of a stochastic predatorprey model with Crowley-Martin functional response, Journal of Applied Mathematics, 2013, pp479-490.

[3] Crowley, PH, Martin, EK. Functional responses and interference within and between year classes of a dragonfly population. J. North Am. Benthol. Soc. 1989, pp211-221.

[4] Xu S. Global stability of the viral dynamics with Crowley-Martin functional response, Electronic Journal of Qualitative Theory of Differential Equations, 2012, pp1-10.

[5] Zhou X and Cui J. Global stability of the viral dynamics with CrowleyMartin functional response, Bull. Korean Math. Soc., 2011, pp555-574.

[6] Khasminskii. R. Stochastic stability of differential equations. second ed. Springer-Verlag, Berlin Heidelberg, 2012.

[7] Mao XR. Stochastic differential equations and application. Horwood Publishing, Chichester, 2007. 